\title{
Quantum Macrostatistical Picture of Nonequilibrium Steady States
}

\author{
by Geoffrey L. Sewell* \\ Department of Physics, Queen Mary, University of London \\ Mile End Road, London E1 4NS, UK
}

\begin{abstract}
We apply our quantum macrostatistical treatment of irreversible processes [18] to prove that, in nonequilibrium steady states, (a) the hydrodynamical observables execute a generalised Onsager-Machlup [12] process and (b) the spatial correlations of these observables are generically of long range. The key assumptions behind these results are a nonequilibrium version of Onsager regression hypothesis [11], together with certain hypotheses of chaoticity and local equilibrium for hydrodynamical fluctuations.
\end{abstract}

Mathematics Subject Classification (2000). 82C10, 82B35, 81R15.

Key Words. quantum macrostatistics, nonequilibrium steady states, chaotic current fluctuations, long range correlations.

\section{Introduction}

It is now well appreciated that a key problem in the statistical mechanics of irreversible processes is the characterisation of nonequilibrium steady states $[2,6,13]$, and a number of different approaches have been made to this problem. Some have employed rather general strategies, based, for example, on a hypothesis of Anosov dynamics [6,13]; while others comprise treatments of concrete microscopic stochastic dynamical models $[20,4,1]$.

A different approach to nonequilibrium statistical mechanics has been made by the present author in a number of works [16-18] that are centred on the hydrodynamical observables of quantum systems. This approach, like Onsager's [11] treatment of the subject, is designed to form a bridge beween the microscopic and macroscopic pictures of matter, rather than a deduction of the latter from the former. Its basic assumptions concern only very general, model-independent properties of many-particle systems, and its scope is thus intended to be complementary to that of works based on microscopic treatments of many-body problems. The results to which it has led [18] include a mathematical characterisation of local thermodynamical equilibrium and a generalisation of Onsager's reciprocity relations to a regime where the macroscopic dynamics is nonlinear.

In this note, we extend the macrostatistical scheme by the introducation of a chaoticity hypothesis for the fluctuations of the non-conserved currents associated with the locally

* E-mail address g.l.sewell@qmul.ac.uk 
conserved hydrodynamical observables in nonequilibrium steady states. On this basis we obtain the results that, in these states,

(a) the fluctuations of the hydrodynamical observables execute a generalised OnsagerMachlup (OM) process [12]; and

(b) the spatial correlations of these observables are generically of long range.

The latter result constitutes a mathematical generalisation of results previously proved for certain special classical stochastic models [20,4,1]. At a heuristic level, similar results concerning long range correlations have also been obtained from Landau's fluctuating hydrodynamics $[8,5]$.

We remark here that the result (b) marks a qualitative difference between equilibrium and nonequilibrium steady states, since the hydrodynamical correlations of the former states are generically of short range*, except at critical points.

\section{The Model}

The Quantum Picture. We take the model to be an $N$-particle quantum system, $\Sigma$, that occupies an open, bounded, connected region, $\Omega_{N}$, of a $d$-dimensional Euclidean space $X$ and is coupled at its surface to an array, $\mathcal{R}$, of reservoirs. We assume that the particle number density, $\nu$, of $\Sigma$ is $N$-independent and that $\Omega_{N}$ is the dilation by a factor $L_{N}$ of a fixed, $N$-independent region $\Omega$ of unit volume. Thus $\Omega_{N}=L_{N} \Omega \equiv\left\{L_{N} x \mid x \in \Omega\right\}$ and $L_{N}=(N / \nu)^{1 / d}$. In a standard way, we represent the observables and states of $\Sigma$ by the self-adjoint operators and density matrices, respectively, in a separable Hilbert space $\mathcal{H}_{N}$. We assume that, as has been established under rather general conditions [14, 22], the composite system $(\Sigma+\mathcal{R})$ evolves to a unique steady state, $\omega_{N}$, as $t \rightarrow \infty$. We denote the expectation value, for this state, of an observable $A$ by $\left\langle\omega_{N} ; A\right\rangle$. We shall assume that all interactions are invariant under space translations and rotations.

We assume that $\Sigma$ has a finite set of linearly independent, extensive, conserved observables $\hat{Q}=\left(\hat{Q}_{1}, \ldots, \hat{Q}_{n}\right)$, which intercommute up to surface effects and are thermodynamically complete [18] in the sense that the states corresponding to pure equilibrium phases are labelled by the expectation values of their global densities in the limit $N \rightarrow \infty$. We denote by $s(q)$ the equilibrium entropy density corresponding to the value $q$ of the global density of $\hat{Q}$ in this limit.

We assume the observables $\hat{Q}$ have locally conserved, position dependent densities $\left(\hat{q}_{1}(x), \ldots, \hat{q}_{n}(x)\right):=\hat{q}(x)$, and we denote their evolutes at time $t$, in the Heisenberg picture of the dynamics of the composite $(\Sigma+\mathcal{R})$, by $\hat{q}^{(N)}(x, t) \equiv \hat{q}_{t}^{(N)}(x)=\left(\hat{q}_{1, t}^{(N)}(x), \ldots, \hat{q}_{n, t}^{(N)}(x)\right)$. In accordance with the standard requirements of quantum field theories [21], we assume that the fields $\hat{q}_{j, t}^{(N)}$ are operator-valued distributions. Thus, denoting by $\mathcal{D}^{\prime}\left(\Omega_{N}\right)$ the space of continuous linear functionals on the subset of the L. Schwartz space $\mathcal{D}(X)[15]$ with

* Our distinction between 'long' and 'short' range will be expressed in a sharp mathematical form in Section 4 
support in $\Omega_{N}$, we assume that the fields $\hat{q}_{t}^{(N)}$ are operator-valued elements of $\mathcal{D}^{\prime}\left(\Omega_{N}\right)^{n}$.

The Hydrodynamical Picture of $\Sigma$. We assume that this is given by a continuum mechanical law governing the evolution of a set of locally conserved classical fields $q_{t}(x)=$ $\left(q_{1, t}(x), ., q_{n, t}(x)\right)$, on a macroscopic space-time scale that we shall presently specify. Here the fields $q_{j, t}(x)$ represent the densities at position $x$ and time $t$ of the extensive thermodynamical variables of $\Sigma$. In general, their dynamics is given by a classical equation of motion of the form

$$
\frac{\partial q_{t}(x)}{\partial t}=\mathcal{F}\left(q_{t} ; x\right)
$$

the right hand side representing the value of a functional $\mathcal{F}$ of $q_{t}$ at the position $x$. For simplicity we base our treatment here on the case where this equation corresponds to a nonlinear diffusion and thus takes the form

$$
\frac{\partial q_{t}}{\partial t}=\nabla \cdot\left(K\left(q_{t}\right) \nabla q_{t}\right)
$$

subject to certain stationary, spatially varying boundary conditions determined by the reservoirs $\mathcal{R}$. Here and elsewhere $q_{t}$ is a function of position, and $K\left(q_{t}\right)$ is an $n$-by- $n$ matrix $\left[K_{j k}\left(q_{t}\right)\right]$, whose action on $\nabla q_{t}$ is given by standard matrix multiplication. We shall presently specify the relationship between $q_{t}$ and the quantum field $\hat{q}_{t}^{(N)}$.

We assume that the unit of the macroscopic length scale is the distance $L_{N}$, introduced above; and correspondingly, since Eq. (1) is invariant under the scale transformations $x \rightarrow \lambda x, t \rightarrow \lambda^{2} t$, we take the macroscopic time scale to be $L_{N}^{2}$. Thus, Eq. (1) represents a phenomenolgical dynamics in the bounded spatial region $\Omega$ on this time scale. We assume that that equation admits a unique stationary solution, $q_{t}=q$, subject to the imposed boundary conditions. We assume throughout that the system is in a single phase region wherein both $K(q)$ and the entropy density $s(q)$ are smooth functions of $q$ as this variable runs over the range of $q(x)$ for $x \in \Omega$.

A simple important consequence of Eq. (1) is that, although the fields $q_{t}$ are locally conserved, the associated currents are not. This is crucial for our key assumption, formulated in Section 3, to the effect that the irreversibility of the flow of $q_{t}$ stems from chaoticity properties of the fluctuations of these currents*.

Relationship between the Quantum and Hydrodynamical Pictures. We assume that the classical field $q$ is the expectation value of $\hat{q}$ for the steady state $\omega_{N}$, as represented on the macroscopic length scale of unit $L_{N}$. Thus,

$$
q(x)=\lim _{N \rightarrow \infty}\left\langle\omega_{N} ; \hat{q}\left(L_{N} x\right)\right\rangle \forall x \in \Omega .
$$

Correspondingly, since the unit of the macroscopic time scale is $L_{N}^{2}$, we represent the timedependent fluctuations of $\hat{q}_{t}(x)(\equiv \hat{q}(x, t))$ about its steady state value, on the macroscopic

* In the case of Navier-Stokes hydrodynamics, it would be the stress tensor, not the mass current, that would be the locally non-conserved field, and our chaoticity assumption would pertain to the fluctuations in this tensor. 
scale, by the field

$$
\xi_{t}^{(N)}(x)=N^{1 / 2}\left[\hat{q}^{(N)}\left(L_{N} x, L_{N}^{2} t\right)-\left\langle\omega_{N} ; \hat{q}\left(L_{N} x\right)\right\rangle\right],
$$

the normalisation parameter $N^{1 / 2}$ being the canonical one for fluctuations [7].

\section{The Fluctuations: a Generalised Onsager-Machlup Process.}

We now assume, on grounds [16-18] representing the intercommutativity of the quantum fields $\hat{q}_{j, t}(x)$ and $\hat{q}_{k, u}(y)$ at macroscopic space-time separation and the fact that the ratio of macroscopic to microscopic relaxation times becomes infinite in the limit $N \rightarrow \infty$, that the quantum process $\xi_{t}^{(N)}$ reduces to a classical stationary Markov process $\xi_{t}$ in this limit, i.e. that

$$
\begin{gathered}
\lim _{N \rightarrow \infty}\left\langle\omega_{N} ; \xi_{t_{1}}^{(N)}\left(x_{1}\right) \otimes \xi_{t_{2}}^{(N)}\left(x_{2}\right) \ldots \otimes \xi_{t_{m}}^{(N)}\left(x_{m}\right)\right\rangle=E\left(\xi_{t_{1}}\left(x_{1}\right) \otimes \xi_{t_{2}}\left(x_{2}\right) \ldots \otimes \xi_{t_{m}}\left(x_{m}\right)\right) \\
\forall x_{1}, \ldots, x_{m} \in \Omega, t_{1}, \ldots, t_{m} \in \mathbf{R}_{+},
\end{gathered}
$$

where $\otimes$ denotes the $\mathbf{R}^{n}$ tensor product. Since $\mathcal{D}^{\prime}$ spaces are complete, it follows from this formula that $\xi_{t}$ is a distribution-valued random field. The forward time derivative of $\xi_{t}$, as defined by Nelson [10], is

$$
D \xi_{t}=\lim _{h \rightarrow+0} h^{-1} E\left(\xi_{t+h}-\xi_{t} \mid \xi_{t}\right),
$$

where $E\left(. \mid \xi_{t}\right)$ denotes the conditional expectation functional, given the field $\xi_{t}$.

Regression Hypothesis. We now invoke a version of Onsager's regression hypothesis [11], to the effect that the regressions of the fluctuations $\xi_{t}$ are governed by the same dynamical law as the 'small' perturbations $\delta q_{t}$ of the macroscopic field $q$. To this end, we start by inferring from Eq. (1) that the linearized equation of motion for $\delta q_{t}$ is

$$
\frac{\partial}{\partial t} \delta q_{t}(x)=\mathcal{L} \delta q_{t}(x):=\nabla \cdot\left(K(q(x)) \nabla\left(\delta q_{t}(x)\right)+\left[K^{\prime}(q(x)) \delta q_{t}(x)\right] \nabla q(x)\right),
$$

where $K^{\prime}(q)$ is the derivative of $K(q)$, i.e. its gradient w.r.t. the variable $q$ : thus $\left[K^{\prime}(q) \delta q_{t}\right]_{j k}=\sum_{l=1}^{n}\left[\partial K_{j k}(q) / \partial q_{l}\right] \delta q_{l, t}$. We assume that the perturbation of $q$ does not change its boundary conditions, i.e. that $\delta q_{t}$ vanishes on the boundary of $\Omega$.

We assume that $\delta q_{t}$, like the quantum field $\hat{q}$, is a $\mathcal{D}^{\prime}(\Omega)^{n}$-class distribution and that the operator $\mathcal{L}$, defined in Eq. (6), is the generator of a one-parameter semigroup of transformations $\left\{T_{t} \mid t \in \mathbf{R}_{+}\right\}$of $\mathcal{D}^{\prime}(\Omega)^{n}$, as determined by the equation

$$
\frac{d T_{t}}{d t}=\mathcal{L} T_{t} ; T_{0}=I
$$

and thus that the solution of Eq. (6) is

$$
\delta q_{t}=T_{t-s} \delta q_{s} \forall t \geq s \geq 0 .
$$


We assume that the system satisfies the dissipativity condition that the perturbation $\delta q_{t}$ vanishes in the limit $t \rightarrow \infty$, i.e. that

$$
\lim _{t \rightarrow \infty} T_{t} \phi=0
$$

for all elements of $\phi$ that vanish on the boundary of $\Omega$.

We now assume, as a generalisation of Onsager's regression hypothesis for equilibrium fluctuations that, for $t \geq s \geq 0$, the conditional expectation of $\xi_{t}$, given $\xi_{s}$, is

$$
E\left(\xi_{t} \mid \xi_{s}\right)=T_{t-s} \xi_{s} \forall t \geq s \geq 0
$$

Hence, by Eqs. (5), (7) and (10),

$$
D \xi_{t}=\mathcal{L} \xi_{t}
$$

Further, by Eq. (10) and the stationarity of the $\xi_{t}$ process, the two-point function $E\left(\xi_{t}(x) \otimes \xi_{t^{\prime}}\left(x^{\prime}\right)\right)$ takes the value $\left(T_{t-t^{\prime}} \otimes I\right) E\left(\xi(x) \otimes \xi\left(x^{\prime}\right)\right)$ or $\left(I \otimes T_{t^{\prime}-t}\right) E\left(\xi(x) \otimes \xi\left(x^{\prime}\right)\right)$ according to whether or not $t \geq t^{\prime}$. Thus,

$$
\begin{gathered}
E\left(\xi_{t}(x) \otimes \xi_{t^{\prime}}\left(x^{\prime}\right)\right)= \\
\left(T_{t-t^{\prime}} \otimes I\right) E\left(\xi(x) \otimes \xi\left(x^{\prime}\right)\right) \theta\left(t-t^{\prime}\right)+\left(I \otimes T_{t^{\prime}-t}\right) E\left(\xi(x) \otimes \xi\left(x^{\prime}\right)\right)\left(1-\theta\left(t-t^{\prime}\right)\right),
\end{gathered}
$$

where $\theta$ is the Heaviside function that takes the value 1 or 0 according to whether or not its argument is non- negative.

Extended Stochastic Process: the Currents. Recalling that the fields $\hat{q}_{t}$ are locally conserved observables, we assume that the above formulation of the stochastic process $\xi_{t}$ has a canonical extension to a larger process $\left(\xi_{t}, \eta_{t}\right)$, where $\eta_{t}$ represents the fluctuations of the currents* associated with $\hat{q}_{t}$. Thus, $\xi_{t}$ and $\eta_{t}$ conform to the local conservation law

$$
\frac{\partial \xi_{t}}{\partial t}+\nabla \cdot \eta_{t}=0
$$

and consequently, by Eqs. (5) and (11),

$$
\mathcal{L} \xi_{t}=-E\left(\nabla \cdot \eta_{t+0} \mid \xi_{t}\right)
$$

We now define

$$
\tilde{\eta}_{t}=\eta_{t}-E\left(\eta_{t+0} \mid \xi_{t}\right)
$$

* Note that it follows from the local conservation laws that the divergences of these currents are also classical. Hence, it is irrelevant for our purposes that the currents themselves might not be classical, since they enter into our calculations only through $\nabla . \eta$. In fact, we have obtained the same results by a fully quantum treatment [19] of the currents, which does not contain formal conditional expectations such as that in Eq. (15). 
and

$$
b_{t}=-\nabla \cdot \tilde{\eta}_{t} \equiv-\frac{\partial}{\partial x_{\mu}} \tilde{\eta}_{\mu, t},
$$

where $x_{\mu}$ and $\eta_{\mu, t}$ are the $\mu^{\prime}$ th Cartesian components of $x$ and $\tilde{\eta}_{t}$, respectively. We note that Eq. (15) may naturally be interpreted as signifying that $E\left(\eta_{t+0} \mid \xi_{t}\right)$ and $\tilde{\eta}_{t}$ are the secular and residual stochastic parts, respectively, of $\eta_{t}$; and thus Eq. (16) signifies that $b_{t}$ is minus the divergence of the stochastic part of the current.

It follows now from Eqs. (13)-(16) that

$$
\frac{d \xi_{t}}{d t}=\mathcal{L} \xi_{t}+b_{t}
$$

which has the form of a Langevin equation*. Our interpretation of it, however, will depend on the chaoticity and local equilibrium hypotheses that we shall presently introduce. At all events, $b_{t}$ is statistically independent of $\xi\left(:=\xi_{0}\right)$ for $t>0$, since, by Eqs. (7), (10) and $(17)$

$$
E\left(b_{t} \mid \xi\right)=\frac{d}{d t} E\left(\xi_{t} \mid \xi\right)-\mathcal{L} E\left(\xi_{t} \mid \xi\right)=\left(\frac{d}{d t}-\mathcal{L}\right) T_{t} \xi=0
$$

Further, by Eq. (17),

$$
E\left(b_{t}(x) \otimes b_{t^{\prime}}\left(x^{\prime}\right)\right)=\left(\frac{\partial}{\partial t}-\mathcal{L} \otimes I\right)\left(\frac{\partial}{\partial t^{\prime}}-I \otimes \mathcal{L}^{\prime}\right) E\left(\xi_{t}(x) \otimes \xi_{t^{\prime}}\left(x^{\prime}\right)\right)
$$

where $\mathcal{L}^{\prime}$ is the image of $\mathcal{L}$, as defined by Eq. (6), under the transformation $x \rightarrow x^{\prime}$. Hence, by Eqs. (7) and (12), together with the identity $d \theta\left(t-t^{\prime}\right) / d t \equiv \delta\left(t-t^{\prime}\right)$,

$$
E\left(b_{t}(x) \otimes b_{t^{\prime}}\left(x^{\prime}\right)\right)=-\left[E\left(\mathcal{L} \xi(x) \otimes \xi\left(x^{\prime}\right)\right)+E\left(\xi(x) \otimes \mathcal{L}^{\prime} \xi\left(x^{\prime}\right)\right)\right] \delta\left(t-t^{\prime}\right) .
$$

The higher order correlation functions of $b_{t}$ will be governed by the following chaoticity hypothesis.

Chaoticity Hypothesis. This is the hypothesis that the space-time correlations of the stochastic part of the non-conserved current fluctuations associated with $\hat{q}_{t}$, as viewed on the microscopic scale, are of short range. Since the ratios of the macroscopic to microscopic scales of both length and time are infinite, this signifies that the space-time correlations of the currents $\tilde{\eta}_{t}(x)$ have zero range. Further, since the fluctuations of quantum fields with short range correlations are generally Gaussian in the large scale limit [7], we assume that $\tilde{\eta}_{t}$ is a Gaussian process. Thus, our chaoticity assumption is that $\tilde{\eta}_{t}$ is a Gaussian field

\footnotetext{
* Strictly speaking, $\tilde{\eta}_{t}$ and $b_{t}$ should be treated as distributions w.r.t. $t$, since it will presently emerge that each of them corresponds to a white noise (cf. Eqs. (18) and (29)). Mathematical propriety can easily be achieved, however, without changing the structure of the argument, by working with the integral $w_{t}=\int_{0}^{t} d u b_{u}$, which would correspond to a Wiener process, instead of $b_{t}$.
} 
with zero range space-time correlations. Hence, by a standard theorem on distributions [15; Theorem 35], we can state the chaoticity hypothesis as follows.

(C) $\tilde{\eta}_{t}$ is a stationary Gaussian process whose two- point function $E\left(\tilde{\eta}_{\mu, t}(x) \otimes \tilde{\eta}_{\nu, t^{\prime}}\left(x^{\prime}\right)\right)$ is a finite linear combination of $\delta\left(x-x^{\prime}\right) \delta\left(t-t^{\prime}\right)$ and its derivatives, with coefficients given by generalised functions of $x$.

We note here that the Gaussian property of $b_{t}$ implies that of $\xi_{t}$, for the following reasons. Since $\mathcal{L}$ is the generator of the semigroup $T$, it follows from Eq.(17) that

$$
\xi_{t+t_{0}}=T_{t+t_{0}} \xi+\int_{0}^{t+t_{0}} d s T_{t+t_{0}-s} b_{s} \forall t, t_{0} \geq 0 .
$$

By $(C)$, the integral on the r.h.s. of this equation is Gaussian, while, by Eq. (9), the first term on its r.h.s. vanishes in the limit $t \rightarrow \infty$. Hence, as the stationarity of the process $\xi_{t}$ implies that it is isomorphic with $\xi_{t+t_{0}}$, it must be Gaussian.

Equilibrium Conditions. As a preliminary to the formulation of local equilibrium conditions for the nonequilibrium steady state, we first formulate the true equilibrium twopoint functions of the fields $\xi_{t}$ and $\eta_{t}$. For this purpose we assume that the equilibrium state is achieved by arranging the reservoirs $\mathcal{R}$ so that $q(x)$ is uniform on the boundary, $\partial \Omega$, of $\Omega$. With this boundary condition, Eq. (1) has a stationary solution in which $q(x)$ is uniform throughout $\Omega$. Consequently, by our uniqueness assumption of Section $2, q(x)$ reduces to a constant, $q$ in the equilibrium state. Further, recalling our assumption of Section 2 that the interactions of the system are translationally and rotationally invariant, we assume that the corresponding symmetries are not broken in the pure equilibrium phase and thus that the process $\left(\xi_{t}, \tilde{\eta}_{t}\right)$ is invariant under the space translations and rotations that are implementable within the confines of $\Omega$. We remark here that the limitation in the Euclidean symmetry imposed by the boundedness of $\Omega$ is not serious from the physical standpoint, since any point of this open region corresponds, in the microscopic picture, to one that is infinitely far from the boundary of the quantum system $\Sigma$.

Under the above assumptions, a quantum statistical treatment of the field $\xi$ yields the following result for the static two- point function for $\xi$ (cf. [18, Ch. 7, Appendix $\mathrm{C}]$ ): in fact, it corresponds to a thermodynamic limiting version of the Einstein formula $P=$ const. $\exp (S)$.

$$
E_{\text {equil }}\left(\xi(x) \otimes \xi\left(x^{\prime}\right)\right)=J(q) \delta\left(x-x^{\prime}\right),
$$

where $E_{\text {equil }}$ is the equilibrium expectation functional and $J(q)$ is minus the inverse matrix of the Hessian of the entropy density function $s(q)$, i.e.

$$
J(q)=-\left[\partial^{2} s(q) / \partial q_{j} \partial q_{k}\right]^{-1}
$$

On the other hand, it follows from the assumptions of translational and rotational invariance that the two-point function of $\tilde{\eta}_{t}(x)$ takes the form

$$
E_{\text {equil }}\left(\tilde{\eta}_{\mu, t}(x) \otimes \tilde{\eta}_{\nu, t^{\prime}}\left(x^{\prime}\right)\right)=G\left(x-x^{\prime}, t-t^{\prime}\right) \delta_{\mu \nu}
$$


where $G$ is a scalar w.r.t. the vector space $X$. Hence, the chaoticity condition $(C)$ reduces here to the following form.

$(C)_{\text {equil }} . G\left(x-x^{\prime}, t-t^{\prime}\right)$ is a finite linear combination, with constant coefficients, of the distribution $\delta\left(x-x^{\prime}\right) \delta\left(t-t^{\prime}\right)$ and its derivatives.

In order to pin down the explicit form of $G$ we now note that, by Eq. (6), the constancy of $q$ implies that $\mathcal{L}$ is simply $K(q) \Delta$, and therefore, by Eqs. (16), (18) and (19), that

$$
\begin{gathered}
E_{\text {equil }}\left(b_{t}(x) \otimes b_{t^{\prime}}\left(x^{\prime}\right)\right)=\frac{\partial^{2}}{\partial x_{\mu} \partial x_{\nu}^{\prime}} E_{\text {equil }}\left(\tilde{\eta}_{\mu, t}(x) \otimes \tilde{\eta}_{\nu, t^{\prime}}\left(x^{\prime}\right)\right) \\
=2[K(q) J(q)]_{\text {sym }} \Delta \delta\left(x-x^{\prime}\right) \delta\left(t-t^{\prime}\right),
\end{gathered}
$$

where, for any matrix $A,[A]_{\text {sym }}$ denotes the arithmetic mean of $A$ and its transpose*. Hence, by Eqs. (21) and (22),

$$
\Delta G\left(x-x^{\prime}, t-t^{\prime}\right)=2[K(q) J(q)]_{s y m} \Delta \delta\left(x-x^{\prime}\right) \delta\left(t-t^{\prime}\right) .
$$

This equation, together with condition $(C)_{\text {equil }}$, fixes the form of $G$ according to the formula

$$
G\left(x-x^{\prime}, t-t^{\prime}\right)=2[K(q) J(q)]_{s y m} \delta\left(x-x^{\prime}\right) \delta\left(t-t^{\prime}\right),
$$

and consequently, by Eq. (21),

$$
E_{\text {equil }}\left(\tilde{\eta}_{\mu, t}(x) \otimes \tilde{\eta}_{\nu, t^{\prime}}\left(x^{\prime}\right)\right)=2[K(q) J(q)]_{s y m} \delta\left(x-x^{\prime}\right) \delta\left(t-t^{\prime}\right) \delta_{\mu \nu}
$$

We now note that, for any positive $\epsilon$ and any $x_{0} \in \Omega$ and $t_{0} \in \mathbf{R}_{+}$, Eqs. (19) and (23) are invariant under the transformations $x \rightarrow x_{0}+\epsilon x, x^{\prime} \rightarrow x_{0}+\epsilon x^{\prime}, \xi \rightarrow \epsilon^{d / 2} \xi$ and $x \rightarrow x_{0}+$ $\epsilon x, \quad x^{\prime} \rightarrow x_{0}+\epsilon x^{\prime}, t \rightarrow t_{0}+\epsilon^{2} t, \quad t^{\prime} \rightarrow t_{0}+\epsilon^{2} t^{\prime}, \quad \tilde{\eta}_{t} \rightarrow \epsilon^{1+d / 2} \tilde{\eta}_{t}$, respectively. Thus they are equivalent to the following formulae.

$$
\epsilon^{d} E_{\text {equil }}\left(\xi\left(x_{0}+\epsilon x\right) \otimes \xi\left(x_{0}+\epsilon x^{\prime}\right)\right)=J(q) \delta\left(x-x^{\prime}\right),
$$

and

$$
\epsilon^{d+2} E_{\text {equil }}\left(\tilde{\eta}_{\mu, t_{0}+\epsilon^{2} t}\left(x_{0}+\epsilon x\right) \otimes \tilde{\eta}_{\nu, t_{0}+\epsilon^{2} t^{\prime}}\left(x_{0}+\epsilon x^{\prime}\right)\right)=2[K(q) J(q)]_{s y m} \delta\left(x-x^{\prime}\right) \delta\left(t-t^{\prime}\right) .
$$

Evidently, if $\epsilon$ is chosen to be 'small', then these equations correspond to local conditions concentrated at the space-time point $\left(x_{0}, t_{0}\right)$.

* In fact, it has been proved in [18] that $K(q) J(q)$ is a symmetric matrix, i.e. that the Onsager reciprocity relations prevail, subject to the assumption of microscopic reversibility. Moreover, this result was extended there to the nonequilibrium situation under the assumption of a certain local equilibrium hypothesis not employed in this note. However, we shall not assume this symmetry here, since it is not needed for our present purposes. 
Local Equilibrium Conditions. This last observation leads us to propose the following local equilibrium conditions for the general stationary nonequililibrium situation.

$$
\lim _{\epsilon \rightarrow 0} \epsilon^{d} E\left(\xi\left(x_{0}+\epsilon x\right) \otimes \xi\left(x_{0}+\epsilon x^{\prime}\right)\right)=J\left(q\left(x_{0}\right)\right) \delta\left(x-x^{\prime}\right)
$$

and

$$
\begin{gathered}
\lim _{\epsilon \rightarrow 0} \epsilon^{d+2} E\left(\tilde{\eta}_{\mu, t_{0}+\epsilon^{2} t}\left(x_{0}+\epsilon x\right) \otimes \tilde{\eta}_{\nu, t_{0}+\epsilon^{2} t^{\prime}}\left(x_{0}+\epsilon x^{\prime}\right)\right)= \\
2\left[K\left(q\left(x_{0}\right)\right) J\left(q\left(x_{0}\right)\right)\right]_{s y m} \delta\left(x-x^{\prime}\right) \delta\left(t-t^{\prime}\right) \delta_{\mu \nu} .
\end{gathered}
$$

The Generalised Onsager-Machlup Process. In view of the chaoticity condition (C), it follows immediately from this last equation that the two-point function for $\tilde{\eta}_{t}$ must take the following form, since the presence of derivatives of $\delta\left(x-x^{\prime}\right) \delta\left(t-t^{\prime}\right)$ would render the l.h.s. of Eq. (27) divergent.

$$
E\left(\tilde{\eta}_{\mu, t}(x) \otimes \tilde{\eta}_{\nu, t^{\prime}}\left(x^{\prime}\right)\right)=2[K(q(x)) J(q(x))]_{s y m} \delta\left(x-x^{\prime}\right) \delta\left(t-t^{\prime}\right) \delta_{\mu \nu}
$$

It follows from this formula and Eq. (16), the two-point function for $b_{t}$ is

$$
E\left(b_{t}(x) \otimes b_{t^{\prime}}\left(x^{\prime}\right)\right)=-2 \nabla \cdot\left([K(q(x)) J(q(x))]_{s y m} \nabla \delta\left(x-x^{\prime}\right)\right) \delta\left(t-t^{\prime}\right) .
$$

Hence, by the Gaussian assumption in $(C), b_{t}$ is a white noise and consequently, by Eq. (17), $\xi_{t}$ executes a generalised Onsager-Machlup process [12]. We remark here that the two-point function for $b_{t}$ given by Eq. (29) is of the same form as that of the noise term in Landau's fluctuating hydrodynamics [9].

\section{Long Range Spatial Correlations.}

The static two-point function for the fluctuation field $\xi$ is

$$
W\left(x, x^{\prime}\right)=E\left(\xi(x) \otimes \xi\left(x^{\prime}\right)\right) \equiv E\left(\xi_{t}(x) \otimes \xi_{t}\left(x^{\prime}\right)\right) \text { by stationarity }
$$

Since the ratio of the macroscopic to microscopic length scale is infinite, short range correlations on the latter scale reduce to zero on the former one. Accordingly, we term the range of the correlations 'short' or 'long' according to whether or not it reduces to zero in the macroscopic picture. Hence our condition for long range correlations is simply that the support of the distribution $W$ does not lie in the domain $\left\{\left(x, x^{\prime}\right) \in \Omega^{2} \mid x=x^{\prime}\right\}$. The following Proposition establishes that the spatial correlations of $\xi$ are generically of long range.

Proposition. Let $\Phi$ be the $n$-by-n matrix- valued functional on the classical field $q$ defined by the formula

$$
\Phi(q ; x)=\Delta[K(q(x)) J(q(x))]_{s y m}+\nabla . \Psi(q ; x)_{s y m}
$$


where

$$
\Psi_{j k}(q ; x)=\left[\frac{\partial}{\partial q_{m}(x)} K_{j l}(q(x))\right]\left[J_{m k}(q(x)) \nabla q_{l}(x)-J_{l k}(q(x)) \nabla q_{m}(x)\right]
$$

Then under the above assumptions, a sufficient condition for the spatial correlations of $\xi$ to be of long range is that $\Phi(q)$ does not vanish.

Comments. (1) The Proposition establishes that the correlations are generically of long range, since the condition that $\Phi(q)$ vanishes can be satified only for special relationships between the functions $K(q(x))$ and $s(q(x))$. By contrast, the corresponding correlations for equilibrium states are generically of short range, except at critical points. A treatment of critical equilibrium correlations of fluctuation observables is provided in Ref. [3].

(2) In the particular case of the symmetric exclusion process $[20,4], n=1, d=$ $1, K(q)=1, s(q)=-q \ln q-(1-q) \ln (1-q)$ and $q(x)=a+b . x$, where $a$ and $b(\neq 0)$ are constants. Thus, in this case, it follows from Eqs. (20), (31) and (32) that $\Phi(q ; x)=$ $-2 b^{2} \neq 0$. Hence, as proved by other methods in Refs. [20, 4], long range correlations prevail in this model.

Proof of Proposition. Suppose that the spatial correlations of $\xi$ are not of long range, i.e. that the support of $W$ does lie in the domain $\left\{\left(x, x^{\prime}\right) \in \Omega^{2} \mid x=x^{\prime}\right\}$. Then it follows from a classic theorem on distributions [15; Theorem 35] that $W\left(x, x^{\prime}\right)$ is a finite linear combination of $\delta\left(x-x^{\prime}\right)$ and its derivatives, with coefficients given by generalised functions of $x$. Under this assumption, it follows from Eqs. (26) and (30), together with the continuity of $J(q(x))$, that this combination must reduce to the form

$$
W\left(x, x^{\prime}\right)=J(q(x)) \delta\left(x-x^{\prime}\right)
$$

since the presence of derivatives of $\delta\left(x-x^{\prime}\right)$ would cause the l.h.s. of Eq. (26) to diverge. On comparing Eqs. (18) and (29), we see that

$$
\left(\mathcal{L} \otimes I+I \otimes \mathcal{L}^{\prime}\right) E\left(\xi(x) \otimes \xi\left(x^{\prime}\right)\right)=2 \nabla \cdot\left([K(q(x)) J(q(x))]_{s y m} \nabla \delta\left(x-x^{\prime}\right)\right) .
$$

Hence, as $a \otimes b$ is the transpose of $b \otimes a$,

$$
\begin{gathered}
{\left[(\mathcal{L} \otimes I) E\left(\xi(x) \otimes \xi\left(x^{\prime}\right)\right)\right]_{s y m}+\left[\left(\mathcal{L}^{\prime} \otimes I\right) E\left(\xi\left(x^{\prime}\right) \otimes \xi(x)\right)\right]_{s y m}=} \\
2 \nabla \cdot\left([K(q(x)) J(q(x))]_{\text {sym }} \nabla \delta\left(x-x^{\prime}\right)\right),
\end{gathered}
$$

i.e., by Eqs. (30) and (33),

$$
\begin{gathered}
{\left[\mathcal{L}\left(J(q(x)) \delta\left(x-x^{\prime}\right)\right)\right]_{s y m}+\left[\mathcal{L}^{\prime}\left(J\left(q\left(x^{\prime}\right)\right) \delta\left(x^{\prime}-x\right)\right)\right]_{s y m}=} \\
2 \nabla \cdot\left([K(q(x)) J(q(x))]_{s y m} \nabla \delta\left(x-x^{\prime}\right)\right) .
\end{gathered}
$$


We now infer from the formula for $\mathcal{L}$ given by Eq. (6), together with Eq. (32), that

$$
\begin{gathered}
\mathcal{L}\left(J(q(x)) \delta\left(x-x^{\prime}\right)\right)= \\
\Delta\left[K(q(x)) J(q(x)) \delta\left(x-x^{\prime}\right)\right]+\nabla \cdot\left[\Psi(q ; x) \delta\left(x-x^{\prime}\right)\right],
\end{gathered}
$$

while the interchange of $x$ and $x^{\prime}$ in this formula yields the equation

$$
\begin{gathered}
\mathcal{L}^{\prime}\left(J\left(q\left(x^{\prime}\right)\right) \delta\left(x^{\prime}-x\right)\right)=\Delta^{\prime}\left[K\left(q\left(x^{\prime}\right)\right) J\left(q\left(x^{\prime}\right)\right) \delta\left(x^{\prime}-x\right)\right]+\nabla^{\prime} \cdot\left[\Psi\left(q ; x^{\prime}\right) \delta\left(x^{\prime}-x\right)\right]= \\
\Delta^{\prime}\left[K(q(x)) J(q(x)) \delta\left(x-x^{\prime}\right)\right]+\nabla^{\prime} \cdot\left[\Psi(q ; x) \delta\left(x-x^{\prime}\right)\right]= \\
K(q(x)) J(q(x)) \Delta \delta\left(x^{\prime}-x\right)-\Psi(q ; x) \cdot \nabla \delta\left(x-x^{\prime}\right) .
\end{gathered}
$$

It now follows easily from the last two equations, together with the definition (31), that the difference between the left and right hand sides of Eq. (36) is simply $\Phi(q ; x) \delta\left(x-x^{\prime}\right)$. This signifies that the assumption of short range correlations implies that $\Phi(q)=0$; or equivalently that a sufficient conditions for the static $\xi$-correlations to be of long range is that $\Phi(q)$ does not vanish.

\section{Concluding Remarks}

The quantum macrostatistical theory presented here is based on the regression hypothesis together with the assumptions of local equilibrium and chaotic current fluctuations. On this physical basis, we have obtained both a generalised Onsager-Machlup process and a generic picture of long range correlations in nonequilibrium steady states for systems whose phenomenological dynamics corresponds to a multi-component nonlinear diffusion. Our derivation of these results depended on a coordination of the spatial and temporal macroscopic scalings, which was facilitated by the invariance of the assumed phenomenological law, given by Eq. (1), under the scale transformation $x \rightarrow \lambda x, t \rightarrow \lambda^{2} t$. We remark that, for systems whose phenomenological laws do not have any simple scale invariance, e.g. for Navier-Stokes hydrodynamics, the situation is clearly much more complex; and a generalisation of our results to such cases would presumably require an intricate multi-scale analysis.

\section{References}

1. Bertini, L., De Sole, A., Gabrielli, D., Jona-Lasinio, G. and Landim, C.: Macroscopic fluctuation theory for stationary nonequilibrium states, J. Stat. Phys. 107 (2002), 635675 .

2. Bonetto, R., Lebowitz, J. L. and Rey-Bellet, L.: Fourier's law: a challenge to theorists: Pp. 128-150, Mathematical Physics 2000, Ed. A. Fokas, A. Grigorian, T. Kibble, B. Zegarlinski, Imperial College Press, 2000.

3. Broidioi, M., Momont, B. and Verbeure, A.: Lie algebra of anomolously scaled fluctuations: J. Math. Phys. 36 (1995), 6746-6757. 
4. Derrida, B., Lebowitz, J. L. amd Speer, E. R.: Large deviation of the density profile in the symmetric simple exclusion process, J. Stat. Phys. 107 (2002), 599-634.

5. Dorfman, J. R., Kirkpatrick, T. R. and Sengers, J. V.: Generic long range correlations in molecular fluids, Annu. Rev. Phys. Chem. 45 (1994), 213-239.

6. Gallavotti, G.: Chaotic hypothesis: Onsager reciprocity and fluctuation-dissipation theorem: J. Stat. Phys. 84 (1996), 899-926.

7. Goderis, D, Vets, P. and Verbeure, A.: Noncommutative central limits, Prob. Th. Re. Fields 82 (1989), 527-544.

8. Grinstein, G., Lee, D.-H. and Sachdev, S.: Conservation laws, anisotropy and "selforganized criticality" in noisy nonequilibrium systems, Phys. Rev. Lett. 64 (1990), 19271930.

9. Landau, L. D. and Lifschitz, E. M.: Fluid Mechanics, Pergamon, Oxford, 1984.

10. Nelson, E.: Dynamical Theories of Brownian Motion, Princeton Univ. Press, Princeton, 1972.

11. Onsager, L.: Reciprocal relations in irreversible processes, Phys. Rev. 37 (1931), 405-426; 38 (1931), 2265-2279.

12. Onsager, L. and Machlup, S.: Fluctuations and irreversible processes, Phys. Rev. 91 (1953), 1505-1512; ibid 1512-1515.

13. Ruelle, D.: Positivity of entropy production in nonequilibrium statistical mechanics, J. Stat. Phys. 85 (1996), 1-25.

14. Ruelle, D.: Natural nonequilibrium states in quantum statistical mechanics, J. Stat. Phys. 98 (2000), 57-75.

15. Schwartz, L.: Théorie des Distributions, Tome 1, Hermann, Paris, 1951.

16. Sewell, G. L.: Quantum macrostatistics and irreversible thermodynmica, Pp. 368-83 of Lecture Notes in Mathematics Vol. 1442, Ed. L.Accardi and W. Von Waldenfels, Springer, Berlin, 1990.

17. Sewell, G. L.: Towards a macrostatistical mechanics, Pp. 130-152 of Mathematical Physics towards the 21st Century, Ed. R. N. Sen and A. Gersten, Ben- Gurion University of the Negev Press, 1994.

18. Sewell, G. L.: Quantum Mechanics and its Emergent Macrophysics, Princeton Univ. Press, Princeton, Oxford, 2002.

19. Sewell, G. L.: In preparation.

20. Spohn, H.: Long range correlations for stochastic lattice gases in a nonequilibrium state, J. Phys. A 16 (1983), 4275-4291.

21. Streater, R. F. and Wightman, A. S.: PCT, Spin and Statistics, and All That, W. A. Benjamin, New York, 1964. 
22. Tasaki, S. and Matsui, T.: Fluctuation theorem, nonequilibrium steady states and the MacLennan-Zubarev ensembles of $L^{1}$-asymptotical abelian $C^{\star}$-dynamical systems, Pp. 100-119 of Fundamental Aspects of Quantum Physics, Ed. L. Accardi and S. Tasaki, World Scientific, 2003; mp-arh 02-533 (2002). 